Bundesgesundheitsbl 2016 · 59:1465-1475 DOI 10.1007/s00103-016-2441-5

Online publiziert: 30 . September 2016

(c) Springer-Verlag Berlin Heidelberg 2016

CrossMark

Johannes Zeiher - Gianni Varnaccia - Susanne Jordan - Cornelia Lange

Abteilung für Epidemiologie und Gesundheitsmonitoring, Fachgebiet 27 „Gesundheitsverhalten“, Robert Koch-Institut, Berlin, Deutschland

\title{
Was sind die Einflussfaktoren kindlicher Adipositas?
}

\section{Eine Literaturübersicht im Rahmen des Projekts „Bevölkerungsweites Monitoring adipositasrelevanter Einflussfaktoren im Kindesalter"}

\section{Hintergrund}

Die Prävention von Adipositas bei Kindern und Jugendlichen ist eine der größten gesundheitspolitischen Herausforderungen des 21. Jahrhunderts [1]. In Deutschland gelten $15 \%$ der Kinder und Jugendlichen als übergewichtig oder adipös [2]. Bereits im Kindes- und Jugendalter können Übergewicht und Adipositas die Gesundheit gefährden und bis ins Erwachsenenalter negative gesundheitliche Auswirkungen entfalten $[3,4]$. Vor allem die ersten Lebensjahre spielen in der Prävention von Übergewicht und Adipositas eine zentrale Rolle, da in dieser Zeit bedeutende Risikofaktoren geprägt werden und zahlreiche Risikofaktoren ausschließlich in dieser Lebensphase vermieden werden können [5]. Um der Verbreitung von Übergewicht und Adipositas im Kindes- und Jugendalter entgegenzuwirken, werden bundesweit zahlreiche Maßnahmen der Prävention und Gesundheitsförderung umgesetzt [6].

Eine systematische und kontinuierlich aktualisierte Zusammenstellung relevanter Informationen über die Einflussfaktoren von Adipositas im Kindesalter in Deutschland ist bisher nicht verfügbar. Aus diesem Anlass wird am Robert Koch-Institut bis Ende 2017 im Rahmen des Projekts „Bevölkerungsweites Monitoring adipositasrelevanter Einflussfaktoren im Kindesalter" (AdiMon) ein entsprechendes Indikatorensystem aufgebaut. AdiMon soll die Einflussfaktoren von Adipositas bei Kindern zwischen 0 und 6 Jahren kontinuierlich abbilden und so Handlungsbedarfe in Politik und Praxis frühzeitig erkennen lassen. Die Fokussierung auf Kinder zwischen 0 und 6 Jahren erfolgt aufgrund der zentralen Bedeutung dieser Lebensphase für die Entwicklung von Adipositas [5]. Perspektivisch soll durch ein kontinuierliches Monitoring die Möglichkeit geschaffen werden, Veränderungen rechtzeitig zu registrieren und so Ansatzpunkte für Maßnahmen der Prävention und Gesundheitsförderung zu identifizieren. Auch eine Nutzung im Rahmen der Evaluation von Angeboten und Maßnahmen ist denkbar. Darüber hinaus kann AdiMon von der (Fach-) Öffentlichkeit und den Medien als Informationsquelle genutzt werden. Dieser Beitrag skizziert das methodische Vorgehen bei der Identifizierung geeigneter Indikatoren und stellt die Ergebnisse einer umfassenden Literaturrecherche $\mathrm{zu}$ Einflussfaktoren kindlicher Adipositas dar, welche die Basis für den Aufbau des Monitorings bildet.

\section{Methode}

Mit dem Ziel, ein möglichst umfassendes System von Indikatoren zu gewährleisten, wurde ein mehrschrittiges Verfahren zur Identifizierung relevanter Einflussfaktoren und geeigneter Indikatoren gewählt (•Abb. 1). Die Recherche bezog sich dabei sowohl auf kindliches Übergewicht als auch aufkindliche Adipositas, da vermieden werden sollte, durch eine zu enge Begriffsdefinition mögliche Einflussfaktoren im Vorhinein auszuschließen. Zudem wurde keine explizite Altersbegrenzung in Jahren vorgenommen, da auch Ergebnisse von Untersuchungen zu älteren Kindern für die anvisierte Zielgruppe von Bedeutung sein können.

\section{Recherche von Modellen}

Zunächst wurde in den wissenschaftlichen Datenbanken Scopus, PubMed und Google Scholar im Zeitraum zwischen Juni und September 2015 eine elektronische Literaturrecherche nach Artikeln mit Erklärungsmodellen von Adipositas durchgeführt, um einen möglichst umfassenden Überblick über adipositasrelevante Einflussbereiche im Kindesalter und das Wechselverhältnis einzelner Einflussfaktoren zu bekommen. Kombiniert wurden die drei Begriffsgruppen Modell (z. B. „conceptual model“), Übergewicht und Adipositas (z. B. „obesity“) und Zielgruppe (z. B. „children“), wobei der Suchterm eine Kombination der Begriffe aus allen drei Gruppen beinhaltete und im Titel oder Abstract der Publikation enthalten sein musste. Die Suche wurde auf einen Veröffentlichungszeitraum von 2006 bis 2015 begrenzt. Weitere Publikationen wurden per Freihandsuche sowie aus den Ergebnissen der separat erfolgten Suche nach Übersichtsarbeiten zu 


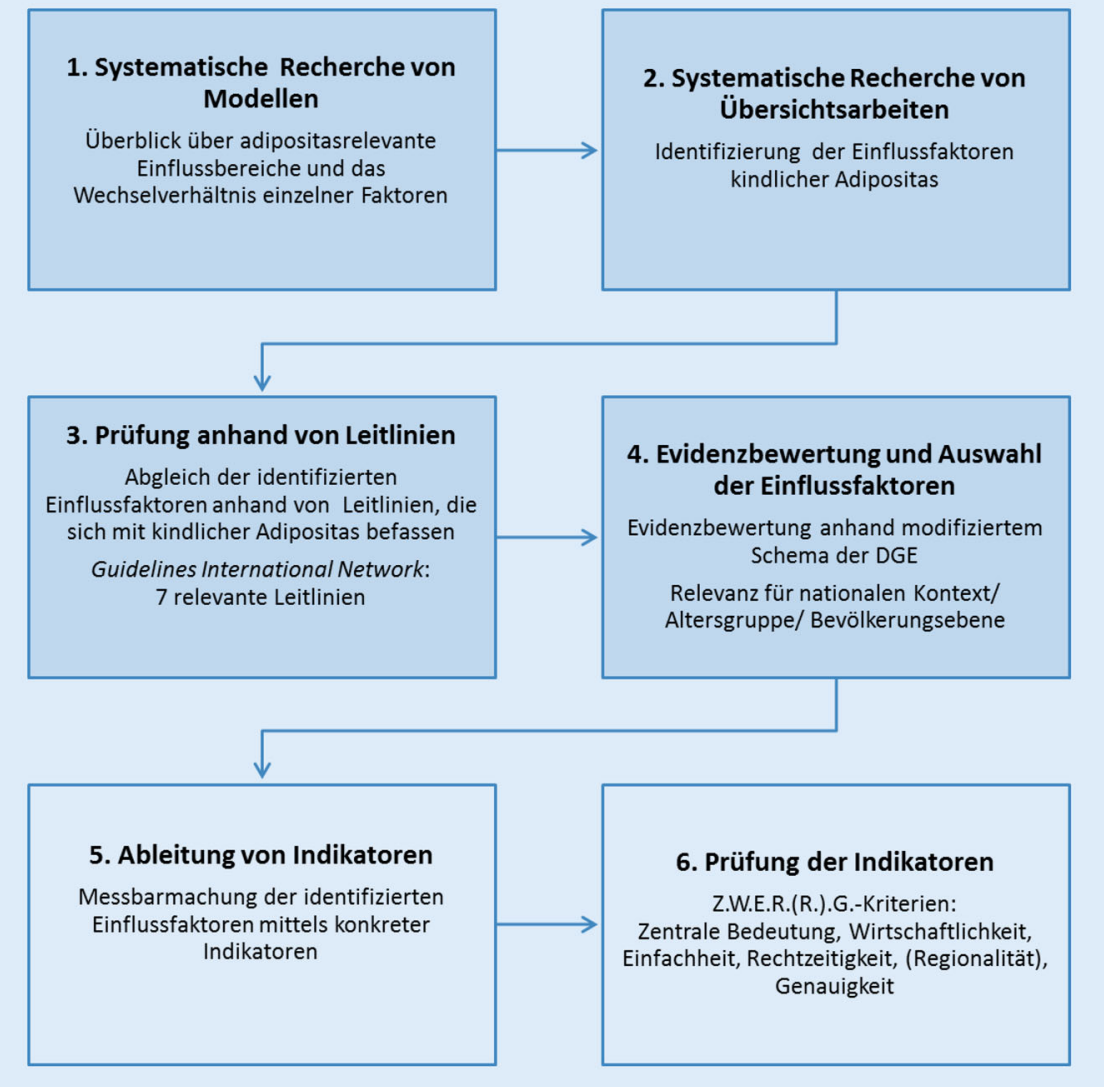

Abb. 1 A Schematische Darstellung des methodischen Vorgehens bei der Entwicklung von Indikatoren für ein nationales Monitoring der Einflussfaktoren kindlicher Adipositas. (Hinweis: Bei den Schritten 1-4 handelt es sich um bereits abgeschlossene Projektschritte, die in dieser Publikation vorgestellt werden.)

Einflussfaktoren von Adipositas im Kindesalter ergänzt (s. • Abb. 2).

\section{Recherche von Übersichtsarbeiten}

Um den aktuellen Wissensstand zu adipositasrelevanten Einflussfaktoren im Kindesalter zusammenzutragen, wurde in den wissenschaftlichen Datenbanken Scopus, PubMed und Google Scholar im Zeitraum zwischen Juli und Dezember 2015 eine elektronische Literaturrecherche nach Übersichtsarbeiten durchgeführt. Die Suchstrategie umfasste die drei Begriffsgruppen Einflussfaktor (z. B. „determinants"), Übergewicht und Adipositas (z. B. „obesity“) und Zielgruppe (z. B. „children“). Den auf den Titel der Publikation begrenzten Suchterm bildete schließlich eine Kombination der drei Begriffsgruppen. Auch hier wurde die Suche auf einen Veröffentlichungszeitraum von 2006 bis 2015 begrenzt.
Weitere Publikationen wurden per Freihandsuche ergänzt (s. - Abb. 2). Die Ergebnisse wurden vom Erstautor nach Einflussbereichen gegliedert, in eine Übersichtsmatrix überführt und durch den Zweitautor bestätigt.

\section{Recherche von Leitlinien}

Im nächsten Schritt wurde die Vollständigkeit der ermittelten Einflussfaktoren anhand von internationalen Leitlinien, die sich mit Einflussfaktoren kindlicher Adipositas befassen, geprüft. Dies sollte gewährleisten, dass in der Liste der ermittelten Einflussfaktoren keine thematischen Lücken übersehen werden. Relevante Leitlinien wurden auf der elektronischen Datenbank „Guidelines International Network" (http://www.g-in.net/library/international-guidelineslibrary) unter Verwendung des Suchbegriffs „obesity“ identifiziert (s. ๑ Abb. 2).

\section{Auswahl von Einflussfaktoren}

Mit Blick auf das zu entwickelnde bevölkerungsweite Monitoring wurden die identifizierten Einflussfaktoren anschlieBend einem Auswahlprozess unterzogen. Kriterien für den Ein- bzw. Ausschluss waren die Bedeutung auf Bevölkerungsebene, die Relevanz für die Altersgruppe (0-6 Jahre) und die Bedeutung im nationalen Kontext. Im nächsten Schritt erfolgte eine Evidenzbewertung der Einflussfaktoren. Hierbei wurden die den identifizierten Übersichtsarbeiten zugrunde liegenden Studienergebnisse sowie Angaben zur Studienart extrahiert und anhand des angepassten Evidenzbewertungsschemas der Deutschen Gesellschaft für Ernährung beurteilt (s. DTab. 1).

Anschließend wurden alle Einflussfaktoren ausgeschlossen, die in der Mehrzahl der in den Übersichtsarbeiten berücksichtigten Studien keinen Zusammenhang mit der Entstehung von Adipositas im Kindesalter zeigen. Einflussfaktoren, bei denen die Evidenzbewertung eine „unzureichende Studienlage“ ergab, wurden berücksichtigt, um neuere Forschungsergebnisse mit bislang wenigen Studien nicht auszuschließen. Teilweise wurden zusätzlich Einzelstudien herangezogen, um genauere Informationen über Einflussfaktoren zu gewinnen, die in den identifizierten Modellen, Übersichtsarbeiten und Leitlinien nur knapp beschrieben werden. Die Ergebnisse der Recherche wurden auf einem Expertenworkshop im Februar 2016 diskutiert und abgestimmt.

\section{Ergebnisse}

Die Suche nach Erklärungsmodellen von Adipositas im Kindesalter erzielte 21 relevante Treffer (s. DTab. 2). Die identifizierten Erklärungsmodelle verdeutlichen die Bedeutung einer sozialökologischen Perspektive. Dabei wird das Individuum im Kontext externer Faktoren betrachtet, die auf verschiedenen Ebenen wirken und teilweise miteinander interagieren [8]. Das Verhalten einer Person ist somit nicht allein das Resultat individueller Faktoren wie Einstellungen, Motivation und Selbststeuerung (Volition), 
Bundesgesundheitsbl 2016·59:1465-1475 DOI 10.1007/s00103-016-2441-5

๑) Springer-Verlag Berlin Heidelberg 2016

\section{J. Zeiher · G. Varnaccia · S. Jordan · C. Lange}

\section{Was sind die Einflussfaktoren kindlicher Adipositas? Eine Literaturübersicht im Rahmen des Projekts „Bevölkerungsweites Monitoring adipositasrelevanter Einflussfaktoren im Kindesalter"}

\section{Zusammenfassung}

Hintergrund. Bereits im Kindesalter kann

Adipositas die Gesundheit gefährden und bis ins Erwachsenenalter negative gesundheitliche Auswirkungen entfalten. Eine systematisierte und kontinuierlich aktualisierte Zusammenstellung relevanter Informationen über die multifaktoriellen Ursachen kindlicher Adipositas ist in Deutschland bisher nicht verfügbar. Der Beitrag stellt die Ergebnisse einer systematischen Literaturrecherche nach Einflussfaktoren kindlicher Adipositas dar, die im Zuge des Aufbaus eines bevölkerungsweiten Monitorings durchgeführt wurde.

Methode. Die Suchstrategie bestand aus drei Schritten: Zunächst wurde die Literatur nach Erklärungsmodellen der kindlichen Adipositas durchsucht. Darauf aufbauend erfolgte eine Recherche von Übersichtsarbeiten zu Einflussfaktoren kindlicher Adipositas. Die Ergebnisse wurden anschließend mit internationalen Leitlinien, die sich mit Einflussfaktoren kindlicher Adipositas befassen, abgeglichen. Ergebnisse. Es wurden 21 Erklärungsmodelle, 75 Übersichtsarbeiten und 7 Leitlinien identifiziert und über 60 Einflussfaktoren der kindlichen Adipositas extrahiert. Diese lassen sich in die Bereiche Ernährungs-, Bewegungs- und Schlafverhalten, biologische Faktoren und Erkrankungen, pränatale und frühkindliche Faktoren, psychosoziale Faktoren, Lebensmittelangebot, Bewegungsmöglichkeiten, Setting und soziales Umfeld, Präventionsmaßnahmen und
Gesundheitsförderung, sozioökonomische, demografische, soziokulturelle und sonstige Faktoren gliedern.

Schlussfolgerungen. Der Beitrag verdeutlicht die komplexe Struktur der Einflussfaktoren kindlicher Adipositas im Sinne eines sozialökologischen Erklärungsansatzes. Die Recherche bildet die Basis für das Monitoringsystem „Bevölkerungsweites Monitoring adipositasrelevanter Einflussfaktoren im Kindesalter", das bis Ende 2017 am Robert Koch-Institut aufgebaut wird.

Schlüsselwörter Adipositas · Einflussfaktoren · Gesundheitsmonitoring $\cdot$ Kinder $\cdot$ Prävention

\section{What are the determinants of childhood obesity? A literature review as part of the project "Nationwide Monitoring of Childhood Obesity Determinants"}

\section{Abstract}

Background. Obesity can impair health even in childhood and unfold negative health consequences through an individual's lifespan. In Germany, to date, a systematic and periodically updated synopsis of the multifaceted determinants of childhood obesity is lacking. In this paper, we present the results of a systematic literature review on childhood obesity determinants, which was conducted over the course of the implementation of nationwide monitoring. Methods. The review was carried out in three steps. Initially, a search for etiological models of childhood obesity was conducted. Based on these results, a systematic review of reviews on childhood obesity determinants was carried out. Finally, the results were verified by taking international guidelines on childhood obesity into account.

Results. In total, 21 etiological models, 75 reviews and 7 guidelines were identified. Over 60 determinants were extracted from these publications and were summarized into the following categories: nutritional behavior, physical activity behavior, sleeping pattern, biological determinants and diseases, prenatal and early childhood determinants, psychosocial determinants, food environment, moveability/walkability, setting and social environment, health promotion and prevention, socioeconomic, demographic, and sociocultural determinants.

Conclusion. This review demonstrates the complex patterns of childhood obesity determinants in correspondence with a socio-ecological approach. The review will form the basis for the monitoring-system "Nationwide Monitoring of Childhood Obesity Determinants", which will be implemented at the Robert Koch Institute by the end of 2017.

Keywords Obesity - Determinants · Health monitoring . Children · Prevention sondern auch das Ergebnis zahlreicher Umweltfaktoren. Zur verbesserten Übersicht können die identifizierten Erklärungsmodelle entsprechend ihrer grafischen Darstellung in Ring- und Flussdiagramme unterteilt werden. Ringdiagramme beschränken sich auf die Darstellung der Einflussebenen und führen teilweise einzelne Einflussfaktoren auf. Flussdiagramme heben hingegen die kausalen Beziehungen und teilweise auch die Wechselwirkungen zwischen einzelnen
Einflussfaktoren und der Entstehung von Adipositas hervor.

Die Suche nach Übersichtsarbeiten zu Einflussfaktoren kindlicher Adipositas erzielte 75 relevante Treffer. Insgesamt wurden über 60 Einflussfaktoren identifiziert, die für die Entstehung von Adipositas im Kindesalter relevant sind und die Kriterien für ein bevölkerungsweites Monitoring erfüllen. Des Weiteren wurden sieben Leitlinien identifiziert, die sich mit der Entstehung von Adipositas im Kindesalter befassen (s. • Tab. 3).
Die identifizierten Leitlinien wurden von medizinischen Fachgesellschaften als klinische Leitlinien verfasst [30, 31], von Adipositas-Fachgesellschaften veröffentlicht [32] oder entstammen PublicHealth-Einrichtungen, die sich mit der Qualitätssicherung im Gesundheitswesen befassen [33-36]. Die Prüfung der Leitlinien bestätigte die Auswahl der bisher identifizierten Einflussfaktoren.

Im Folgenden werden die im Rahmen der Literaturrecherche identifizierten und für das bevölkerungsweite 

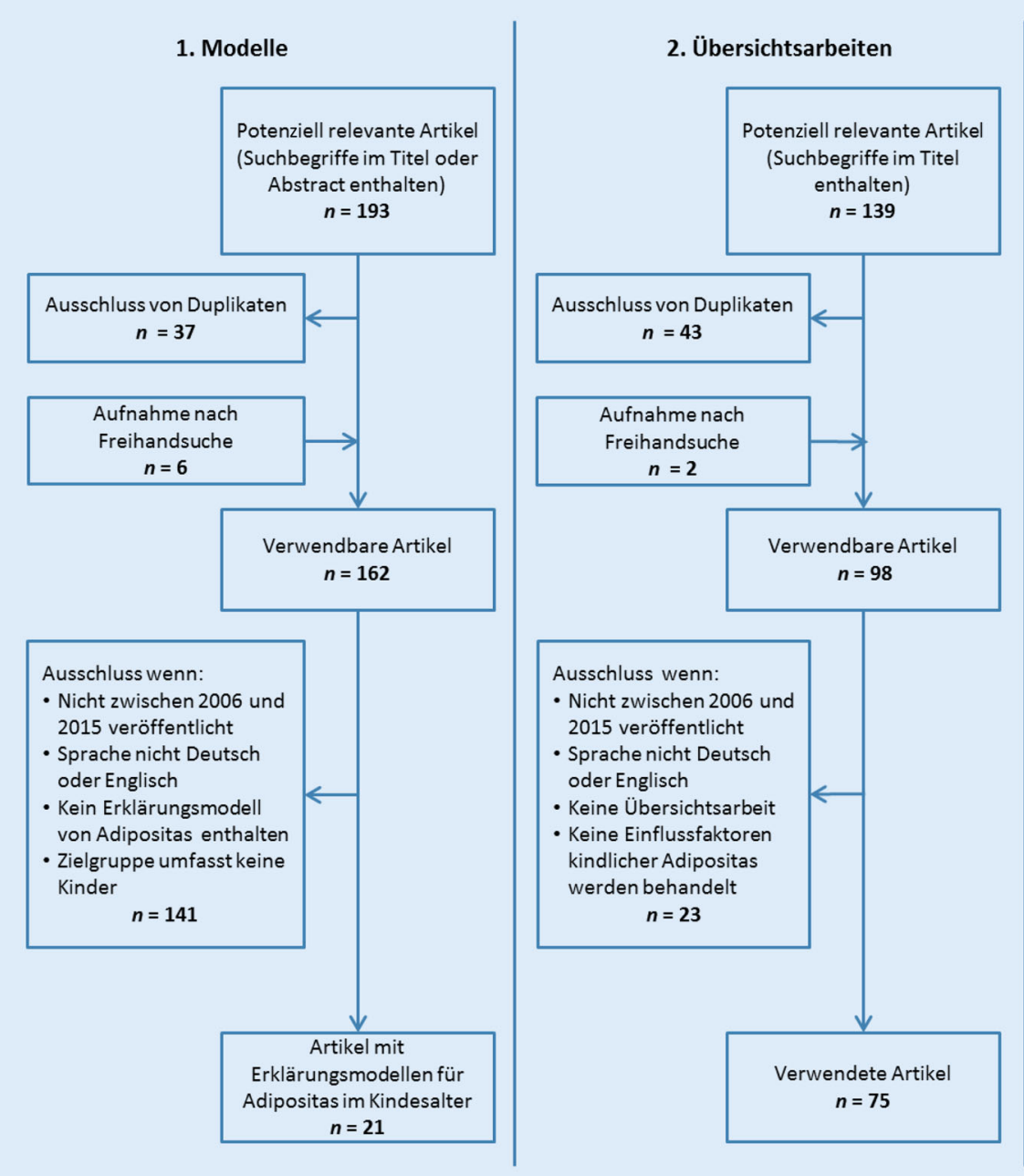

\section{Leitlinien}

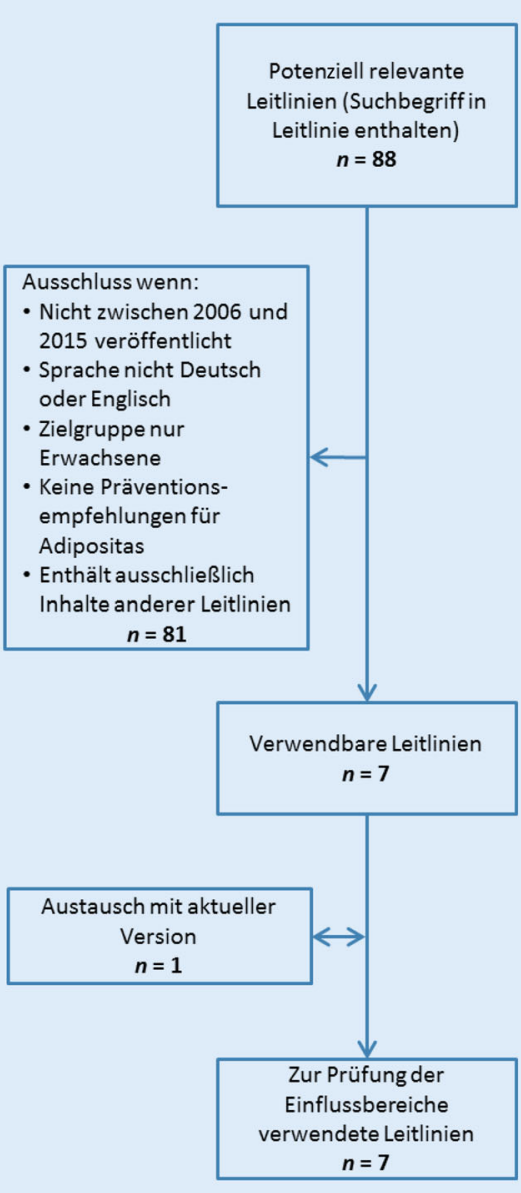

Abb. 2 ॥ Selektionsprozess der Übersichtsarbeiten, Leitlinien und Modelle, die für die Auswertung der Einflussfaktoren der Adipositas im Kindesalter herangezogen wurden

Monitoring zu berücksichtigenden Einflussfaktoren von Adipositas im Kindesalter vorgestellt. Für die Darstellung der Ergebnisse werden die Einflussfaktoren in thematische Bereiche eingeteilt. Einflussfaktoren der Adipositas sind individuelle Verhaltensweisen (Ernährung, körperliche [In-]Aktivität, Schlaf) sowie biologische Faktoren, wie die genetische Disposition oder das Geburtsgewicht. Das Gesundheitsverhalten wird darüber hinaus mit psychosozialen Faktoren wie der Ausprägung der Selbstregulation in Verbindung gebracht. Zudem sind bestimmte Lebensphasen für die Entstehung der kindlichen Adipositas von besonderer Bedeutung. Neben diesen Faktoren wird das adipositasrelevante Verhalten von Verhältnisfaktoren (z. B. Lebensmittelverfügbarkeit, Erreichbar- keit von Bewegungsmöglichkeiten) und Angebotsfaktoren wie Präventionsmaßnahmen in Kitas beeinflusst. Kontextfaktoren wie der sozioökonomische Status wirken auf allen Ebenen auf die Entstehung kindlicher Adipositas ein.

\section{Ernährung}

Wenngleich empirische Untersuchungen keine eindeutigen Befunde liefern, gilt eine erhöhte Energieaufnahme als zentraler Risikofaktor für die Entstehung von Adipositas im Kindesalter [37-39]. Konsistente Studienergebnisse liegen für Lebensmittel mit einer hohen Energiedichte (Energiegehalt pro Gewichtseinheit) vor, deren häufiger Verzehr die Entstehung von Adipositas im Kindesalter begünstigt [40]. Auch ein häufiger Konsum zuckerhaltiger Erfrischungsgetränke [37, 39] sowie große Portionsgrößen [41-43] und ein häufiger Verzehr von Fast Food $[39,44]$ fördern die Entstehung von Adipositas im Kindesalter. Nicht eindeutig stellt sich die Studienlage beim Verzehr von Snacks [37, 39], Obst und Gemüse [37, 45] sowie bei Mustern in der Nährstoffverteilung dar [37-39]. Einen Einfluss auf die Entstehung von Adipositas bei Kindern hat auch das Essverhalten: Bestimmte Mahlzeitenmuster wie ein regelmäßiges Frühstück oder das gemeinsame Essen mit der Familie beugen der Entstehung von Adipositas im Kindesalter vor [39, 46-49]. Des Weiteren ist auffälliges Essverhalten wie „emotional eating" oder „binge eating" mit Adipositas im Kindesalter assoziiert $[50,51]$. 
Tab. 1 Schema der Evidenzbewertung (mod. nach DGE 2015) [7]

$\begin{array}{ll}\text { Evidenzklasse } & \text { Art der Studie } \\ \text { I } & \text { Interventionsstudien } \\ \text { II } & \text { Kohortenstudien } \\ \text { III } & \text { Fall-Kontroll-Studien } \\ \text { IV } & \text { Nicht analytische Studien (z. B. Querschnittstudien) }\end{array}$

\section{Härtegrad der Aussage}

Überzeugende

Evidenz

Mindestens $2 \mathrm{im}$ Ergebnis übereinstimmende Studien der Evidenzklasse I oder mindestens 5 Studien der Evidenzklasse II und Nachweis der biologischen Plausibilität

Ausreichende

Evidenz

Die Mehrzahl der vorliegenden Studien (mindestens 3) muss in eine Richtung weisen

Unzureichende

Studienlage
Es gibt wenige Studien, die eine Assoziation zwischen einem Einflussfaktor und dem Outcome andeuten, aber zu dessen Etablierung unzureichend sind

\section{Körperliche (In-)Aktivität und Schlaf}

Auf der „anderen Seite“ der Energiebilanz - beim Energieverbrauch - spielt die körperliche Aktivitätbzw. Inaktivität eine wichtige Rolle. Bezogen auf die allgemeine körperliche Aktivität (d. h. ohne Differenzierung von sportlicher, Freizeit- und Transportaktivität) zeigt sich in prospektiven Studien ein inverser Zusammenhang mit kindlichem Übergewicht und Adipositas [37, 38]. Eine häufige sitzende Betätigung - oft erfasst über die Dauer des Fernsehkonsums - begünstigt die Entstehung von Übergewicht und Adipositas im Kindesalter [38, 52]. Mehrere systematische Übersichtsarbeiten zeigen, dass eine kürzere Schlafdauer mit einem deutlich höheren Adipositasrisiko - vor allem in jungen Jahren - einhergeht [38, 53].

\section{Biologische Faktoren und Erkrankungen}

Unter dem Einflussbereich der biologischen Faktoren lassen sich genetische und hormonelle Bedingungen zusammenfassen, die mit der Entstehung von Adipositas im Kindesalter in Verbindung gebracht werden. Belegt ist, dass eine genetische Veranlagung der Eltern für Adipositas einen signifikanten Risikofaktor für die Entstehung von Adipositas im Kindesalter darstellt [37, 54]. Des Weiteren stehen bestimmte Hormone [37, 55] und Bakterien im Darmtrakt [39, 55] im Verdacht, Adipositas ggf. mitverursachen zu können. Auch be- völkerungsweit relevante Erkrankungen (z. B. Asthma) sind mit Adipositas im Kindesalter assoziiert [3, 36].

\section{Pränatale und frühkindliche Faktoren}

Für die Entwicklung kindlicher Adipositas sind bestimmte kritische Lebensphasen von besonderer Bedeutung. Eine solche kritische Phase ist der Zeitraum vor der Geburt des Kindes. Eine hohe Gewichtszunahme der Mutter während der Schwangerschaft (unabhängig vom absoluten Gewicht) begünstigt die Entstehung einer Adipositas des Kindes im späteren Lebensverlauf [56]. Gleiches gilt für das Rauchen der Mutter während der Schwangerschaft [57, 58] und mütterlichen Diabetes bzw. Schwangerschaftsdiabetes [59, 60]. Ein hohes Geburtsgewicht ist mit der späteren Entwicklung einer Adipositas assoziiert [57]. Auch eine schnelle Gewichtszunahme im ersten Lebensjahr begünstigt die Entstehung von Adipositas im Kindesalter [57, 61]. Stillen hat eine protektive Funktion bei der Entstehung von kindlicher Adipositas, wobei eine längere Stilldauer den positiven Effekt verstärken soll [57, 62]. Für einen frühen Zeitpunkt der Einführung [57] und die Zusammensetzung der Beikost [63] ist die Studienlage nicht eindeutig. Die Art und Weise, wie ein Kleinkind oder Säugling ernährt wird (beispielsweise ein „emotionaler“ oder ein durch Beschränkungen charakterisierter Fütterungsstil), kann einen Einfluss auf das Körpergewicht und die Entstehung von Adipositas haben $[64,65]$.

\section{Psychosoziale Faktoren}

Individuelle Merkmale der Kinder wie eine geringe Selbstregulation [66], ein geringes Selbstbewusstsein [67] und ein geringes Ausmaß an sozialen Ressourcen [68] werden mit einer höheren Wahrscheinlichkeit für kindliche Adipositas in Verbindung gebracht. Für den Einfluss von Stress - sowohl bei Kindern als auch bei Eltern - liegen keine eindeutigen Befunde vor [55, 69]. Auch eine psychische Erkrankung der Eltern (hier: der Mutter), wie bespielweise eine Depression, ist mit kindlicher Adipositas assoziiert [70]. Sowohl die eigene Körperwahrnehmung [23] als auch die Körperwahrnehmung durch die Eltern [71] kann sich - wenn das Körperbild als problematisch angesehen wird - auf das Ernährungs- und Bewegungsverhalten der Kinder auswirken und somit die Entstehung von Adipositas fördern. Weitere psychosoziale Einflussfaktoren sind eine ausgeprägte Sensibilität gegenüber Außenreizen (z. B. gegenüber Essensgeruch) [72], sowie eine geringe Gesundheitskompetenz der Eltern $[73,74]$ und des Kindes [75], wobei die Studienlage hier als unzureichend $\mathrm{zu}$ bewerten ist.

\section{Lebensmittelangebot}

Weniger auf der individuellen als auf der Verhältnisebene (d.h. bei den Lebens- und Umweltbedingungen) sind Untersuchungen anzusiedeln, die sich mit dem Lebensmittelangebot beschäftigen. Hierbei werden unter anderem das verfügbare Lebensmittelangebot im Lebensraum von Kindern [76, 77] sowie die Kennzeichnung von Mahlzeiten und Lebensmitteln [78, 79] als Determinanten des Ernährungsverhaltens diskutiert. Darüber hinaus zeigen Übersichtsarbeiten einen Zusammenhang zwischen dem Lebensmittelpreis und dem elterlichen Kaufverhalten [80, 81]. Abgesehen davon steht die Nährstoffzusammensetzung von Lebensmitteln, z. B. ein hoher Fett- oder Zuckeranteil, mit einer Gewichtszunahme bei Kindern in Verbindung [82, 83]. 
Tab. 2 Ergebnisse der Suche nach Modellen zur Erklärung von Adipositas im Kindesalter

\begin{tabular}{|c|c|c|c|c|}
\hline Autoren & Bezeichnung des Modells & Diagrammtyp & Jahr & Quelle \\
\hline Zhou und Chea & $\begin{array}{l}\text { Ecological Risk Model of Childhood } \\
\text { Obesity in Chinese Immigrant Child- } \\
\text { ren }\end{array}$ & Ringdiagramm & 2015 & [9] \\
\hline Schmidt et al. & $\begin{array}{l}\text { Conceptual Model for Relationships } \\
\text { between Neighborhood Charac- } \\
\text { teristics and BMI Development in } \\
\text { Children }\end{array}$ & Flussdiagramm & 2015 & {$[10]$} \\
\hline $\begin{array}{l}\text { Ohri-Vachaspati } \\
\text { et al. }\end{array}$ & $\begin{array}{l}\text { Social Ecological Model Showing the } \\
\text { Layers Influencing a Child's Weight } \\
\text { Status }\end{array}$ & Ringdiagramm & 2014 & {$[11]$} \\
\hline Ayala et al. & $\begin{array}{l}\text { Conceptual Model for SOL Youth: } \\
\text { Understanding Risk and Protective } \\
\text { Factors for Latino Childhood Obesity }\end{array}$ & Ringdiagramm & 2014 & {$[12]$} \\
\hline Davison et al. & The Original Family Ecological Model & Sonstiger Typ & 2013 & {$[13]$} \\
\hline Pallan et al. & $\begin{array}{l}\text { Schematic Diagram of UK South } \\
\text { Asian Community Stakeholders' Per- } \\
\text { ceived Causes of Childhood Obesity }\end{array}$ & Flussdiagramm & 2012 & {$[14]$} \\
\hline Harrist et al. & $\begin{array}{l}\text { Interpersonal and Intrapersonal Risk } \\
\text { Model of Child Obesity }\end{array}$ & Flussdiagramm & 2012 & {$[15]$} \\
\hline Zeeb et al. & $\begin{array}{l}\text { Einflussfaktoren auf das kindliche } \\
\text { Gewicht und Ansatzpunkte für pri- } \\
\text { märe Prävention und Gesundheits- } \\
\text { förderung }\end{array}$ & Flussdiagramm & 2011 & {$[16]$} \\
\hline Williams et al. & $\begin{array}{l}\text { Hypothetical Social-Ecological Mo- } \\
\text { del Illustrating Family and Broader } \\
\text { Environmental Influences on Child } \\
\text { Obesity }\end{array}$ & Flussdiagramm & 2011 & {$[17]$} \\
\hline Reed et al. & $\begin{array}{l}\text { Expanded Version of the Davison and } \\
\text { Birch Ecological Model of Predictors } \\
\text { of Childhood Overweight }\end{array}$ & Ringdiagramm & 2011 & {$[18]$} \\
\hline Pinard et al. & Model of the Home Environment & Flussdiagramm & 2011 & {$[19]$} \\
\hline Harrison et al. & $\begin{array}{l}\text { The Six-C's Developmental Ecological } \\
\text { Model of Contributors to Overweight } \\
\text { and Obesity in Childhood }\end{array}$ & Ringdiagramm & 2011 & {$[20]$} \\
\hline Reisch und Gwozdz & $\begin{array}{l}\text { Die vier Ebenen für gesundheitsrele- } \\
\text { vantes Verhalten bei Kindern }\end{array}$ & Ringdiagramm & 2010 & {$[21]$} \\
\hline Monasta et al. & $\begin{array}{l}\text { The Complex Web of Potential Deter- } \\
\text { minants of Overweight and Obesity } \\
\text { in Children }\end{array}$ & Flussdiagramm & 2010 & {$[22]$} \\
\hline Schneider et al. & $\begin{array}{l}\text { Qualitatives Ursache-Wirkungs-Mo- } \\
\text { dell der Einflussfaktoren auf und } \\
\text { Auswirkungen von Übergewicht/ } \\
\text { Adipositas }\end{array}$ & Flussdiagramm & 2009 & {$[23]$} \\
\hline Lytle & $\begin{array}{l}\text { Conceptual Model: Etiology of Child- } \\
\text { hood Obesity }\end{array}$ & Flussdiagramm & 2009 & {$[24]$} \\
\hline Kumanyika et al. & $\begin{array}{l}\text { Societal Policies and Processes Influ- } \\
\text { encing the Population Prevalence of } \\
\text { Obesity }\end{array}$ & Flussdiagramm & 2008 & {$[25]$} \\
\hline Tabacchi et al. & Determinants of Childhood Obesity & Flussdiagramm & 2007 & {$[26]$} \\
\hline Butland et al. & Obesity System Influence Diagram & Flussdiagramm & 2007 & {$[27]$} \\
\hline Hawkins und Law & Influences on Childhood Overweight & Ringdiagramm & 2006 & {$[28]$} \\
\hline Davison und Birch ${ }^{a}$ & $\begin{array}{l}\text { Ecological Model of Predictors of } \\
\text { Childhood Overweight }\end{array}$ & Ringdiagramm & 2001 & {$[29]$} \\
\hline
\end{tabular}

\section{Bewegungsmöglichkeiten}

Die Verfügbarkeit von Bewegungsmöglichkeiten wie Parks und Spielplätzen im näheren Umfeld und die bewegungsfreundliche Gestaltung des kindlichen Wohnumfeldes („moveability“), bzw. die Möglichkeit, Wege zu Fuß zurückzulegen (,walkability“), wirken sich über die Förderung der körperlichen Aktivität auf das kindliche Körpergewicht aus [68, 84-86]. Kosten, die für Bewegungsangebote entstehen, können ebenfalls eine Rolle spielen, beispielweise Mitgliedsbeiträge für Sportvereine oder die Eintrittspreise für Schwimmbäder [87, 88]. Einschränkend muss angemerkt werden, dass es sich dabei teilweise um Einzelstudien handelt.

\section{Setting und soziales Umfeld}

In Bezug auf den Einfluss der Familie sind in erster Linie die Eltern zu nennen. Ihr Verhalten, beispielsweise durch eine nur moderate Nutzung elektronischer Medien, eine ausgewogene Ernährung oder durch regelmäßige sportliche Aktivität, kann sich positiv auf das Bewegungs- und Ernährungsverhalten ihrer Kinder auswirken und so der Entstehung von Adipositas entgegenwirken $[64,89,90]$. Hinsichtlich der Art und Weise, wie Eltern ihre Kinder erziehen, begünstigt ein autoritärer Erziehungsstil die Entwicklung von kindlicher Adipositas, während ein autoritativer Erziehungsstil eine protektive Wirkung entfaltet $[91,92]$. Die Studienlage ist hier jedoch unzureichend. Die Familienstruktur (beispielweise alleinerziehende Eltern oder das Vorhandensein von Geschwistern) wird ebenfalls als Einflussfaktor diskutiert [93]. In Bezug auf den Haushalt erhöht der Medienzugang den Medienkonsum von Kindern und begünstigt somit die Entstehung von Adipositas [94]. Neben der Familie spielt die Kita im Leben von Kindern eine zentrale Rolle. Hier zeigen Studien, dass eine gesundheitsförderliche Umgebung (beispielsweise ausreichend Bewegungsmöglichkeiten und eine vielfältiges Ernährungsangebot) der Entstehung von Adipositas entgegenwirken kann [95]. Nicht nur der Einfluss der Eltern und der Kita werden als Einflussfaktoren der Adiposi- 
Tab. 3 Ergebnisse der Suche nach Leitlinien zur Prävention von Adipositas im Kindesalter

\begin{tabular}{|c|c|c|c|}
\hline Institution & Leitlinie & Jahr & Quelle \\
\hline $\begin{array}{l}\text { National Institute for Health and } \\
\text { Care Excellence (NICE) }\end{array}$ & $\begin{array}{l}\text { Maintaining a Healthy Weight and Pre- } \\
\text { venting Excess Weight Gain among Adults } \\
\text { and Children }\end{array}$ & 2015 & [33] \\
\hline $\begin{array}{l}\text { Registered Nurses' Association } \\
\text { of Ontario (RNAO) }\end{array}$ & Primary Prevention of Childhood Obesity & 2014 & {$[30]$} \\
\hline $\begin{array}{l}\text { Deutsche Adipositas Gesell- } \\
\text { schaft (DAG) }\end{array}$ & Prävention und Therapie der Adipositas & 2014 & {$[32]$} \\
\hline $\begin{array}{l}\text { Institute for Clinical Systems } \\
\text { Improvement (ICSI) }\end{array}$ & $\begin{array}{l}\text { Prevention and Management of Obesity } \\
\text { for Children and Adolescents }\end{array}$ & 2013 & {$[36]$} \\
\hline $\begin{array}{l}\text { National Health and Medical } \\
\text { Research Council (NHMRC) }\end{array}$ & $\begin{array}{l}\text { Clinical Practice Guidelines for the Man- } \\
\text { agement of Overweight and Obesity in } \\
\text { Adults, Adolescents and Children in Aus- } \\
\text { tralia }\end{array}$ & 2013 & {$[35]$} \\
\hline $\begin{array}{l}\text { Scottish Intercollegiate Guide- } \\
\text { lines Network (SIGN) }\end{array}$ & Management of Obesity & 2010 & {$[34]$} \\
\hline $\begin{array}{l}\text { National Association of Pediatric } \\
\text { Nurse Practitioners (NAPNAP) }\end{array}$ & $\begin{array}{l}\text { Clinical Practice Guideline: Identifying } \\
\text { and Preventing Overweight in Childhood }\end{array}$ & 2006 & [31] \\
\hline
\end{tabular}

tas in Betracht gezogen. Auch das soziale Netzwerk von Freunden und Gleichaltrigen (Peers) kann von Bedeutung sein. So ist ein hohes Level an körperlicher Aktivität im Freundeskreis mit einem hohen individuellen Aktivitätslevel assoziiert, wobei der Zusammenhang für ältere Kinder und Jugendliche beschrieben wurde [96].

\section{Maßnahmen der Adipositas- prävention und Gesundheits- förderung}

Systematische Übersichtsarbeiten zeigen, dass primärpräventive Interventionen positive Effekte auf das Ernährungsund Bewegungsverhalten von Kindern haben können, wohingegen die Studienlage in Bezug aufEffekte von Maßnahmen auf anthropometrische Maße nicht eindeutig ist [95, 97-99]. Ein Großteil der identifizierten Studien bezieht sich auf Interventionen in Betreuungseinrichtungen, wobei auch Maßnahmen unter Einbezug der Eltern oder in Familien untersucht wurden $[95,99]$. Beispiele für Maßnahmen, die in den Übersichtsarbeiten berücksichtigt wurden, sind Informationskampagnen über das Stillen, unstrukturierte Bewegungseinheiten in Kitas (z. B. durch die Erhöhung der täglichen Spielzeit), strukturierte Bewegungseinheiten (z. B. Tanzangebote), ernährungsbezogene Wissensvermittlung in Kitas, die Bereitstellung von Obst und
Gemüse oder Wasserspendern, Informationsveranstaltungen für Eltern oder die Einrichtung eines Kita-Gartens. Als Merkmale für erfolgreiche Maßnahmen werden unter anderem eine Beteiligung der Eltern [95, 98], die Kombination von Ernährungs- und Bewegungsinhalten, ein bestehender Theoriebezug oder die aktive Beteiligung der Kinder angeführt [98].

\section{Sozioökonomische und demografische Faktoren}

In Industrienationen wie Deutschland sind Übergewicht und Adipositas bei Kindern mit einem niedrigem sozioökonomischen Status deutlich weiter verbreitet als in den höheren Statusgruppen [100, 101]. Darüber hinaus werden die Arbeitslosigkeit der Mutter und eine Vollzeit-Erwerbstätigkeit der Eltern (insbesondere der Mutter) als Einflussfaktoren kindlicher Adipositas diskutiert [102, 103]. Die Studienlage ist hierbei allerdings widersprüchlich.

\section{Soziokulturelle Faktoren}

Der kulturelle Hintergrund, unter dem sich Aspekte wie die Religionszugehörigkeit oder Religiosität, die spezifische gesellschaftlich-kulturelle Sichtweise auf Übergewicht und Adipositas, kulturell geprägte Ernährungsgewohnheiten und Ernährungstraditionen wie beispielswei- se das Fasten oder das gemeinsame Familienessen zusammenfassen lassen, spielt bei der Entstehung von kindlicher Adipositas eine Rolle [104]. Einen Einflussfaktor stellt dabei der Migrationshintergrund oder die Migrationserfahrung dar, wobei hier neben den bisher genannten Punkten auch die Anpassungsvorgänge an eine neue Kultur bzw. Gesellschaft als mögliche Ursachen für Unterschiede bei der Verbreitung von Adipositas und Übergewicht diskutiert werden [104-107]. Im Kontext kultureller Faktoren wird auf die Bedeutung des geltenden Schönheitsideals verwiesen, das sowohl die Sichtweise auf abweichende Körperformen als auch das Verhalten beeinflusst, das für den Versuch, dieses Ideal zu erreichen, gezeigt wird (beispielsweise durch Diäten) [50].

\section{Sonstige Faktoren}

In experimentellen Studien wurde beobachtet, dass das Ernährungswissen, die Konsumpräferenzen, das Kaufverhalten sowie das Ernährungsverhalten von Kindern durch Werbung beeinflusst werden können [108-110]. Dabei wird der Kontakt mit Werbung durch eine hohe Nutzungsdauer von Bildschirmmedien begünstigt. Des Weiteren wird die Exposition mit unterschiedlichen chemischen Substanzen wie z. B. Bisphenol A, das unter anderem in Verpackungsmaterial für Lebensmittel enthalten sein kann, mit der Entstehung von Adipositas in Verbindung gebracht [111]. Vermutet wird, dass die hormonaktive Substanz den Fettstoffwechsel beeinflusst.

\section{Diskussion}

Für den Aufbau des bevölkerungsweiten Monitorings wurde eine umfassende Literaturrecherche durchgeführt, um den aktuellen Wissensstand über adipositasrelevante Einflussfaktoren im Kindesalter zusammenzutragen. Die Ergebnisse der Literaturrecherche zeigen, dass neben einer energiereichen Ernährung oder mangelnder körperlicher Aktivität auch Faktoren aus anderen Bereichen eine wichtige Rolle spielen und beim Aufbau eines bevölkerungsweiten Monitorings zu berücksichtigen sind. So ist ne- 
ben dem Ernährungs- und Bewegungsverhalten beispielsweise auch das Schlafverhalten von Bedeutung. Eine weitere Rolle nehmen biologische Faktoren wie die genetische Prädisposition und psychosoziale Faktoren wie bestimmte individuelle Merkmale oder soziale Ressourcen ein. Des Weiteren heben zahlreiche Studien die Bedeutung der Verhältnisse und des unmittelbaren Lebensumfeldes hervor - sei es beim Zugang zu Grünanlagen oder bei der Preisentwicklung bestimmter Lebensmittel. Auch Kontextfaktoren wie der sozioökonomische Status oder der kulturelle Hintergrund haben sich als relevant für die Entwicklung von Adipositas im Kindesalter erwiesen. Im Hinblick auf bestimmte Lebensphasen, die für die Entstehung von Adipositas im Kindesalter von Bedeutung sind, kommt der Schwangerschaft und der frühen Kindheit eine zentrale Rolle zu.

Eine umfassende Literaturrecherche, wie sie in der vorliegenden Arbeit beschrieben wurde, ist mit mehreren Limitationen verbunden. Zunächst ist die hohe Heterogenität der eingeschlossenen Publikationen zu nennen, beispielweise hinsichtlich der untersuchten Altersgruppe. Auf einen restriktiven Ausschluss von Publikationen, die sich nicht explizit auf 0- bis 6-Jährige beziehen, wurde jedoch bewusst verzichtet, da dies einen Großteil der Übersichtsarbeiten ausgeschlossen hätte. Auch die untersuchten Outcome-Variablen unterscheiden sich: Neben verschiedenen anthropometrischen Maßen, wie dem Body-Mass-Index oder der Waist-toHip-Ratio (Taille-Hüft-Quotienten) werden teilweise auch adipositasrelevante Verhaltensweisen als Outcome-Variable untersucht. Des Weiteren wurde bei der Recherche nach adipositasrelevanten Einflussfaktoren im Kindesalter deutlich, dass die Studienlage in den Einflussbereichen sehr unterschiedlich ausfällt. Während es für verhaltensbezogene Einflussfaktoren wie dem Konsum zuckerhaltiger Getränke, dem Stillen oder dem Bewegungsverhalten zahlreiche prospektive Studien und Übersichtsarbeiten mit überzeugender Evidenz gibt, liegen für andere Einflussbereiche, wie z. B. bei psychosozialen Einflussfaktoren, häufig nur wenige oder vorwiegend aus Quer- schnittstudien stammende Ergebnisse vor. Auch im Bereich der adipositasbezogenen Maßnahmen der Prävention und Gesundheitsförderung ist die Studienlage unzureichend: Hier zeigt sich, dass in Deutschland bisher keine umfassende Interventionsberichterstattung existiert, mit der bestehende Angebote in Bezug auf kindliche Adipositas und deren Inanspruchnahme entsprechend quantifiziert werden könnten $[112,113]$. Um potenziell relevante, aber bisher nur unzureichend erforschte Einflussfaktoren nicht aus dem Blick zu verlieren, wurden sie in der Auswahl für das bevölkerungsweite Monitoring berücksichtigt. Eine weitere Limitation stellt die Beschränkung auf Übersichtsarbeiten und - innerhalb der Suchstrategie in den Datenbanken - die Begrenzung des Suchstrings auf den Titel der Publikationen dar. Dies war erforderlich, da eine weitergehende Recherche die Kapazitäten des Projektes überschritten hätte. Trotz des beträchtlichen Umfangs wurde eine eigenständige und aktuelle Recherche aber als notwendig erachtet, um einen umfassenden und aktuellen Überblick über adipositasrelevante Einflussfaktoren im Kindesalter zu ermöglichen. Dies wäre beispielsweise bei Übernahme der Einflussfaktoren aus einer einzelnen Leitlinie nicht der Fall gewesen, da sich diese teilweise nur auf bestimmte Einflussbereiche beschränken (z. B. verhaltensbezogene Einflussfaktoren [33, 34]). Um einen umfassenden Überblick über adipositasrelevante Einflussfaktoren im Kindesalter zu gewährleisten, erfolgte ein Abgleich der in den Modellen, Übersichtsarbeiten und Leitlinien identifizierten Einflussfaktoren.

Die bei AdiMon zusammengestellte Übersicht verdeutlicht die komplexe Struktur der Einflussfaktoren kindlicher Adipositas im Sinne eines sozialökologischen Erklärungsansatzes. Dieser betrachtet das Individuum im Kontext externer Faktoren, die sich auf individuelle Einflussfaktoren auswirken, und lenkt den Blick somit auch auf die Verhältnisse und Lebenswelten, in denen Kinder aufwachsen.

\section{Ausblick}

In der nächsten Projektphase werden die identifizierten und für das bevölkerungsweite Monitoring als relevant eingestuften Einflussfaktoren kindlicher Adipositas in Indikatoren überführt und die gebildeten Indikatoren mit geeigneten $\mathrm{Da}$ tenquellen verknüpft. Darauf aufbauend wird eine Pilotdatensammlung erfolgen, um die gebildeten Indikatoren mit Daten zu hinterlegen bzw. bestehende Datenlücken aufzuzeigen. Perspektivisch werden die gebildeten und mit Daten hinterlegten Indikatoren auf einer internetbasierten Plattform der Öffentlichkeit zugänglich gemacht.

\section{Korrespondenzadresse}

\section{J. Zeiher}

Abteilung für Epidemiologie und Gesundheitsmonitoring, Fachgebiet 27 "Gesundheitsverhalten", Robert Koch-Institut General-Pape-Str. 62-66, 12101 Berlin, Deutschland

ZeiherJ@rki.de

Danksagung. Diese Arbeit wurde unterstützt durch Förderungen des Bundesministeriums für Gesundheit (Förderkennzeichen 2515KIG004).

\section{Einhaltung ethischer Richtlinien}

Interessenkonflikt. J. Zeiher, G. Varnaccia, S. Jordan und C. Lange geben an, dass kein Interessenkonflikt besteht.

Dieser Beitrag beinhaltet keine von den Autoren durchgeführten Studien an Menschen oder Tieren.

\section{Literatur}

1. World Health Organisation (WHO) (2015) Health topics - Noncommunicable diseases - Obesity. http://www.euro.who.int/en/health-topics/ noncommunicable-diseases/obesity. Zugegriffen: 3. Juli 2015

2. Kurth B, Rosario AS (2007) Die Verbreitung von Übergewicht und Adipositas bei Kindern und Jugendlichen in Deutschland. Bundesgesundheitsblatt Gesundheitsforschung Gesundheitsschutz 50(5-6):736-743

3. Pulgaron ER (2013) Childhood obesity: a review of increased risk for physical and psychological comorbidities. Clin Ther 35(1):A18-A32

4. Park MH, Falconer C, Viner RM, Kinra S (2012) The impact of childhood obesity on morbidity and mortality in adulthood: a systematic review. Obes Rev 13(11):985-1000 
5. Birch LL, Ventura AK (2009) Preventing childhood obesity: What works? Int J Obes (Lond) 33(Suppl 1):74-81

6. IN FORM (2015) Deutschlands Initiative für gesunde Ernährung und mehr Bewegung. https:// www.in-form.de.Zugegriffen: 3. Juli 2015

7. Deutsche Gesellschaft für Ernährung (DGE) (2015) Fettzufuhr und Prävention ausgewählter ernährungsmitbedingter Krankheiten. Deutsche Gesellschaft für Ernährung e. V., Bonn

8. Richard L, Gauvin L, Raine K (2011) Ecological models revisited: their uses and evolution in health promotion over two decades. Annu Rev Public Health 32:307-326

9. Zhou N, Cheah CSL (2015) Ecological risk model of childhood obesity in chinese immigrant children. Appetite 90:99-107

10. Schmidt SC, Sleddens EFC, de Vries SI, Gubbels J, Thijs C (2015) Longitudinal association of neighborhood variables with body mass index in dutch school-age children: the KOALA birth cohort study. Soc Sci Med 135:99-108

11. Ohri-VachaspatiP,DeLiaD,DeWeese RS,CrespoNC, Todd M, Yedidia MJ (2014) The relative contribution of layers of the social ecological model to childhood obesity. Public Health Nutr 18(11):2055-2066

12. Ayala GX, Carnethon M, Arredondo E, Delamater AM, Perreira K, Van Horn L et al (2014) Theoretical foundations of the study of latino (SOL) youth: implications for obesity and cardiometabolic risk. Ann Epidemiol 24(1):36-43

13. Davison KK, Jurkowski JM, Lawson HA (2013) Reframing family-centred obesity prevention using the family ecological model. Public Health Nutr 16(10):1861-1869

14. Pallan M, Parry J, Adab P (2012) Contextual influences on the development of obesity in children: a case study of UK south asian communities. Prev Med 54(3-4):205-211

15. Harrist AW, Topham GL, Hubbs-Tait L, Page MC, Kennedy TS, Shriver LH (2012) What developmental science can contribute to a transdisciplinary understanding of childhood obesity: an interpersonal and intrapersonal risk model. Child Dev Perspect 6(4):445-455

16. Zeeb H, Ahrens W, Pigeot I (2011) Primärprävention: Konzepte und Strategien. Bundesgesundheitsblatt Gesundheitsforschung Gesundheitsschutz 54(3):265-271

17. Williams JE, Kabukuru A, Mayo R, Griffin SF (2011) Commentary: a social-ecological perspective on obesity among latinos. Ethn Dis 21(4):467-472

18. ReedDB, Patterson PJ, Wasserman N (2011) Obesity in rural youth: looking beyond nutrition and physical activity. J Nutr Educ Behav 43(5):401-408

19. Pinard CA, Yaroch AL, Hart MH, Serrano EL, McFerren MM, Estabrooks PA (2012) Measures of the home environment related to childhood obesity: a systematic review. Public Health Nutr 15(1):97-109

20. Harrison K, Bost KK, McBride BA, Donovan SM Grigsby-Toussaint DS, Kim J et al (2011) Toward a developmental conceptualization of contributors to overweight and obesity in childhood: the six-Cs model. Child Dev Perspect 5(1):50-58

21. Reisch LA, Gwozdz W (2010) Einfluss des Konsumverhaltens auf die Entwicklung von Übergewicht bei Kindern. Ein Überblick. Bundesgesundheitsblatt Gesundheitsforschung Gesundheitsschutz 53(7):725-732

22. Monasta L, Batty GD, Cattaneo A, Lutje V, Ronfani L, Van Lenthe FJ et al (2010) Early-life determinants of overweight and obesity: a review of systematic reviews. Obes Rev 11(10):695-708
23. Schneider K, Wittig F, Mertens E, Hoffmann I (2009) Qualitatives Ursache-Wirkungs-Modell de Einflussfaktoren auf und Auswirkungen von Übergewicht/Adipositas. http://www.uni-giessen.de/ fbr09/nutr-ecol/_down_pdf/Adipositasmodell_ Endversion_deutsch.pdf. Zugegriffen: 21. Juli 2015

24. Lytle LA (2009) Examining the etiology of childhood obesity: the IDEA study. Am J Community Psychol 44(3-4):338-349

25. Kumanyika SK, Obarzanek E, Stettler N, Bell R, Field AE, Fortmann SP et al (2008) Populationbased prevention of obesity: the need for comprehensive promotion of healthful eating, physical activity, and energy balance: a scientific statement from american heart association council on epidemiology and prevention, interdisciplinary committee for prevention (formerly the expert panel on population and prevention science). Circulation 118(4):428-464

26. Tabacchi G, Giammanco S, La Guardia M, Giammanco $M$ (2007) A review of the literature and a new classification of the early determinants of childhood obesity: from pregnancy to the first years of life. Nutr Res 27(10):587-604

27. Butland B, Jebb S, Kopelman P, McPherson K, Thomas S, Mardell Jetal (2007) Foresight-tackling obesities: future choices. Government Office for Science, London

28. Hawkins SS, Law C (2006) A review of risk factors for overweight in preschool children: a policy perspective. Int J Pediatr Obes 1(4):195-209

29. Davison KK, Birch LL (2001) Childhood overweight: a contextual model and recommendations for future research. Obes Rev 2(3):159-171

30. Registered Nurses' Association of Ontario (RNAO) (2014) Primary prevention of childhood obesity. Clinical best practice guidelines. http://rnao.ca/ sites/rnao-ca/files/Childhood obesity FINAL 19.12.2014.pdf.Zugegriffen: 18 . April 2016

31. National Association of Pediatric Nurse Practitioners (NAPNAP) (2006) Healthy Eating and Activity Together (HEAT) clinical practice guideline: identifying and preventing overweight in childhood. J Pediatr Health Care 20(2):2-32

32. Deutsche Adipositas Gesellschaft (DAG) (2014) Interdisziplinäre Leitlinie der Qualität S3 zur „Prävention und Therapie der Adipositas".http://www. awmf.org/uploads/tx_szleitlinien/050-001I_S3_ Adipositas_Pr\%C3\%A4vention_Therapie_201411.pdf.Zugegriffen: 18 . April 2016

33. National Institute for Health and Care Excellence (NICE) (2015) Maintaining a healthy weight and preventing excess weight gain among adults and children. NICE guideline. http://www.nice.org.uk/ guidance/ng7.Zugegriffen: 18. April 2016

34. Scottish Intercollegiate Guidelines Network (SIGN) (2010) Management of obesity. A national clinical guideline. SIGN, Edinburgh

35. National Health and Medical Research Council (NHMRC) (2013) Clinical practice guidelines for the management of overweight and obesity in adults, adolescents and children in Australia. NHMRC, Melbourne

36. Fitch A, Fox C, Bauerly K, Gross A, Heim C, JudgeDietz J et al (2013) Prevention and management of obesity for children and adolescents. Institute for Clinical Systems Improvement (ICSI), Bloomington

37. Pate RR, O'Neill JR, Liese AD, Janz KF, Granberg EM, Colabianchi N et al (2013) Factors associated with development of excessive fatness in children and adolescents: a review of prospective studies. Obes Rev 14(8):645-658
38. Te Velde $S$, Van Nassau F, Uijtdewilligen L, Van Stralen M, Cardon G, De Craemer M et al (2012) Energy balance-related behaviours associated with overweight and obesity in preschool children: a systematic review of prospective studies. Obes Rev 13(s1):56-74

39. Agostoni C, Braegger C, Decsi T, Kolacek S, Koletzko B, Mihatsch W et al (2011) Role of dietary factors and food habits in the development of childhood obesity: a commentary by the ESPGHAN committee on nutrition. J Pediatr Gastroenterol Nutr 52(6):662-669

40. Perez-Escamilla R, Obbagy JE, Altman JM, Essery EV, McGrane MM, Wong YP et al (2012) Dietary energy density and body weight in adults and children: a systematic review. J Acad Nutr Diet 112(5):671-684

41. Fisher JO, Kral TV (2008) Super-size me: portion size effects on young children's eating. Physiol Behav 94(1):39-47

42. Pourshahidi LK, Kerr MA, McCaffrey TA, Livingstone MB (2014) Influencing and modifying children's energy intake: the role of portion size and energy density. Proc Nutr Soc 73(3):397-406

43. Small L, Lane H, Vaughan L, Melnyk B, McBurnett D (2013) A systematic review of the evidence: the effects of portion size manipulation with children and portion education/training interventions on dietary intake with adults. Worldviews Evid Based Nurs 10(2):69-81

44. Rosenheck R (2008) Fast food consumption and increased caloric intake: a systematic review of a trajectory towards weight gain and obesity risk. Obes Rev 9(6):535-547

45. Ledoux T, Hingle M, Baranowski T (2011) Relationship of fruit and vegetable intake with adiposity: a systematic review. Obes Rev 12(5):e143-e150

46. Kaisari P, Yannakoulia M, Panagiotakos DB (2013) Eating frequency and overweight and obesity in children and adolescents: a meta-analysis. Pediatrics 131(5):958-967

47. Szajewska H, Ruszczynski M (2010) Systematic review demonstrating that breakfast consumption influences body weight outcomes in children and adolescents in Europe. Crit Rev Food Sci Nutr 50(2):113-119

48. Valdes J, Rodriguez-Artalejo F, Aguilar L, JaenCasquero MB, Royo-Bordonada MA (2013) Frequency of family meals and childhood overweight: a systematic review. Pediatr Obes 8(1):e1-e13

49. Hammons AJ, Fiese BH (2011) Is frequency of shared family meals related to the nutritional health of children and adolescents? Pediatrics 127(6):e1565-e1574

50. Russell-Mayhew S, McVey G, Bardick A, Ireland A (2012) Mental health, wellness, and childhood overweight/obesity. J Obes 2012:281801

51. Puder JJ, Munsch S (2010) Psychological correlates of childhood obesity. Int J Obes 34(Suppl 2):37-43

52. Tanaka C, Reilly JJ, Huang WY (2014) Longitudinal changes in objectively measured sedentary behaviour and their relationship with adiposity in children and adolescents: systematic review and evidence appraisal. Obes Rev 15(10):791-803

53. Patel SR, Hu FB (2008) Short sleep duration and weight gain: a systematic review. Obesity (Silver Spring) 16(3):643-653

54. Silventoinen K, Rokholm B, Kaprio J, Sorensen TI (2010) The genetic and environmental influences on childhood obesity: a systematic review of twin and adoption studies. Int J Obes 34(1):29-40

55. Skelton JA, Irby MB, Grzywacz JG, Miller G (2011) Etiologies of obesity in children: nature and nurture. Pediatr Clin North Am 58(6):1333-1354 
56. Lau EY, Liu J, Archer E, McDonald SM, Liu J (2014) Maternal weight gain in pregnancy and risk of obesity among offspring: a systematic review. JObes 2014:524939

57. Weng SF, Redsell SA, Swift JA, Yang M, Glazebrook CP (2012) Systematic review and meta-analyses of risk factors for childhood overweight identifiable during infancy. Arch Dis Child 97(12):1019-1026

58. Riedel C, Schonberger K, Yang S, Koshy G, Chen YC, Gopinath B et al (2014) Parental smoking and childhood obesity: higher effect estimates for maternal smoking in pregnancy compared with paternal smoking - a meta-analysis. Int JEpidemiol 43(5):1593-1606

59. Kim SY, England JL, Sharma JA, Njoroge T (2011) Gestational diabetes mellitus and risk of childhood overweight and obesity in offspring: a systematic review. Exp Diabetes Res 2011:541308

60. Huang JS, Lee TA, Lu MC (2007) Prenatal programming of childhood overweight and obesity. Matern Child Health J 11(5):461-473

61. Druet C, Stettler N, Sharp S, Simmons RK, Cooper C, Smith GD et al (2011) Prediction of childhood obesity by infancy weight gain: an individuallevel meta-analysis. Paediatr Perinat Epidemiol 26(1):19-26

62. Yan J, Liu L, Zhu Y, Huang G, Wang PP (2014) The association between breastfeeding and childhood obesity: a meta-analysis. BMC Public Health 14(1):1267

63. Pearce J, Langley-Evans SC (2013) The types offood introduced during complementary feeding and risk of childhood obesity: a systematic review. Int J Obes 37(4):477-485

64. Larsen JK, Hermans RCJ, Sleddens EFC, Engels RCME, Fisher JO, Kremers SSPJ (2015) How parental dietary behavior and food parenting practices affect children's dietary behavior. Interacting sources of influence? Appetite 89:246-257

65. Bergmeier $\mathrm{H}$, Skouteris $\mathrm{H}$, Hetherington M (2015) Systematic research review of observational approaches used to evaluate mother-child mealtime interactions during preschool years. Am JClin Nutr 101(1):7-15

66. Bergmeier H, Skouteris H, Horwood S, Hooley M, Richardson B (2014) Associations between child temperament, maternal feeding practices and child body mass index during the preschool years: a systematic review of the literature. Obes Rev 15(1):9-18

67. Incledon E, Wake M, Hay M (2011) Psychological predictors of adiposity: systematic review of longitudinal studies. Int J Pediatr Obes 6(2-2):e1-e11

68. Carter MA, Dubois L (2010) Neighbourhoods and child adiposity: a critical appraisal of the literature Health Place 16(3):616-628

69. El-Behadli AF, Sharp C, Hughes SO, Obasi EM, Nicklas TA (2015) Maternal depression, stress and feeding styles: towards a frameworkfor theory and research in child obesity. Br J Nutr 113(S1):S55-S71

70. Benton PM, Skouteris H, Hayden M (2015) Does maternal psychopathology increase the risk of preschooler obesity? A systematic review. Appetite 87:259-282

71. Towns N, D'Auria J (2009) Parental perceptions of their child's overweight: an integrative review of the literature. J Pediatr Nurs 24(2):115-130

72. Braet C (2005) Psychological profile to become and to stay obese. Int J Obes Relat Metab Disord 29(S2):19-23

73. Cha E, Besse JL (2015) Low parent health literacy is associated with "obesogenic" infant care behaviours. Evid Based Nurs 18(2):46
74. Yin HS, Sanders LM, Rothman RL, Shustak R, Eden SK, Shintani A etal (2014) Parenthealth literacyand "obesogenic" feeding and physical activity-related infant care behaviors. J Pediatr 164(3):577-583.e1

75. Chari R, Warsh J, Ketterer T, Hossain J, Sharif I (2014) Association between health literacy and child and adolescent obesity. Patient Educ Couns 94(1):61-66

76. Engler-Stringer R, Le $H$, Gerrard A, Muhajarine $\mathrm{N}$ (2014) The community and consumer food environment and children's diet: a systematic review. BMC Public Health 14:522

77. Pearson N, Biddle SJ, Gorely T (2009) Family correlates of fruit and vegetable consumption in children and adolescents: a systematic review. Public Health Nutr 12(02):267-283

78. Storcksdieck genannt Bonsmann S, Wills JM (2012) Nutrition labeling to prevent obesity: reviewing the evidence from Europe. Curr Obes Rep 1(3):134-140

79. Brambila-Macias J, Shankar B, Capacci S, Mazzocchi M, Perez-Cueto FJ, Verbeke W et al (2011) Policy interventions to promote healthy eating: a review of what works, what does not, and what is promising. Food Nutr Bull 32(4):365-375

80. Rao M, Afshin A, Singh G, Mozaffarian D (2013) Do healthier foods and diet patterns cost more than less healthy options? A systematic review and meta-analysis. BMJOpen 3(12):e004277

81. Kersting M, Claussen K (2007) Wie teuer ist eine gesunde Ernährung für Kinder und Jugendliche? Die Lebensmittelkosten der Optimierten Mischkost als Referenz für sozialpolitische Regelleistungen Ernährungsumsch 54(9):508

82. Te Morenga L, Mallard S, Mann J (2013) Dietary sugars and body weight: systematic review and meta-analyses of randomised controlled trials and cohort studies. BMJ346:e7492

83. Hooper L, Abdelhamid A, Moore HJ, Douthwaite W, Skeaff CM, Summerbell CD (2012) Effect of reducing total fat intake on body weight: systematic review and meta-analysis of randomised controlled trials and cohort studies. BMJ345:e7666

84. Galvez MP, Pearl M, Yen IH (2010) Childhood obesity and the built environment: a review of the literature from 2008-2009. Curr Opin Pediatr 22(2):202

85. Dunton GF, Kaplan J, Wolch J, Jerrett M, Reynolds KD (2009) Physical environmental correlates of childhood obesity: a systematic review. Obes Rev 10(4):393-402

86. BuckC, Tkaczick T, Pitsiladis Y, De Bourdehaudhuij I, Reisch L, Ahrens W et al (2015) Objective measures of the built environment and physical activity in children: from walkability to moveability. J Urban Health 92(1):24-38

87. Allender S, Cowburn G, Foster C (2006) Understanding participation in sport and physical activity among children and adults: a review of qualitative studies. Health Educ Res 21(6):826-835

88. Sonneville KR, La Pelle N, Taveras EM, Gillman MW Prosser LA (2009) Economic and other barriers to adopting recommendations to prevent childhood obesity: results of a focus group study with parents. BMC Pediatr 9(1):1-7

89. Loprinzi PD, Cardinal BJ, Loprinzi KL, Lee H (2012) Parenting practices as mediators of child physical activity and weightstatus. Obes Facts 5(3):420-430

90. Xu H, Wen LM, Rissel C (2015) Associations of parental influences with physical activity and screen time among young children: a systematic review. JObes 2015:546925

91. Vollmer RL, Mobley AR (2013) Parenting styles, feeding styles, and their influence on child obesogenic behaviors and body weight. A review. Appetite 71:232-241

92. Berge JM (2009) A review of familial correlates of child and adolescent obesity: What has the 21st century taught us so far? Int J Adolesc Med Health 21(4):457-483

93. Brisbois TD, Farmer AP, McCargar LJ (2012) Early markers of adult obesity: a review. Obes Rev 13(4):347-367

94. Cillero IH, Jago R (2010) Systematic review of correlates of screen-viewing among young children. Prev Med 51(1):3-10

95. Hesketh KD, Campbell KJ (2010) Interventions to prevent obesity in $0-5$ year olds: an updated systematic review of the literature. Obesity (Silver Spring) 18(Suppl 1):27-35

96. Sawka KJ, McCormack GR, Nettel-Aguirre A, Hawe P, Doyle-Baker PK (2013) Friendship networks and physical activity and sedentary behavior among youth: a systematized review. Int J Behav Nutr Phys Act 130(10):1-9

97. Mikkelsen MV, Husby S, Skov LR, Perez-Cueto F (2014) A systematic review of types of healthy eating interventions in preschools. Nutr J 13(1):56

98. Steenbock B, Pischke C, Schönbach J, Pöttgen S, Brand T (2014) Wie wirksam sind ernährungsund bewegungsbezogene primärpräventive Interventionen im Setting Kita? Bundesgesundheitsblatt Gesundheitsforschung Gesundheitsschutz 58:609-619

99. Redsell SA, Edmonds B, Swift JA, Siriwardena AN, Weng S, Nathan D et al (2015) Systematic review of randomised controlled trials of interventions that aim to reduce the risk, either directly or indirectly, of overweight and obesity in infancy and early childhood. Matern Child Nutr 12(1):24-38. doi:10. $1111 /$ mcn.12184

100. Wu S, Ding Y, Wu F, LiR, Hu Y, Hou Jetal (2015) Socioeconomic position as an intervention against overweight and obesity in children: a systematic review and meta-analysis. Sci Rep 5:11354. doi:10. 1038/srep11354

101. Tamayo T, Christian H, Rathmann W (2010) Impact of early psychosocial factors (childhood socioeconomic factors and adversities) on future risk of type 2 diabetes, metabolic disturbances and obesity: a systematic review. BMC Public Health 10:525

102. Mindlin M, Jenkins R, Law C (2009) Maternal employment and indicators of child health: a systematic review in pre-school children in OECD countries. J Epidemiol Community Health 63(5):340-350

103. Gwozdz W, Sousa-Poza A, Reisch LA, Ahrens W, Eiben G, Fernandéz-Alvira MJ et al (2013) Maternal employment and childhood obesity - a European perspective. J Health Econ 32(4):728-742

104. Goulao B, Santos O, Carmo I (2015) The impact of migration on body weight: a review. Cad Saude Publica 31(2):229-245

105. Labree $L J$, van de Mheen $\mathrm{H}$, Rutten FF, Foets $M$ (2011) Differences in overweight and obesity among children from migrant and native origin: a systematic review of the European literature. Obes Rev 12(5):e535-e547

106. Gilbert PA, Khokhar S (2008) Changing dietary habits of ethnic groups in Europe and implications for health. Nutr Rev 66(4):203-215

107. Delavari M, Sonderlund AL, Swinburn B, Mellor D, Renzaho A (2013) Acculturation and obesity among migrant populations in high income countries - a systematic review. BMC Public Health $13: 458$ 
108. Kraak Vl, Story M (2015) Influence of food companies' brand mascots and entertainment companies' cartoon media characters on children's diet and health: a systematic review and research needs. Obes Rev 16(2):107-126

109. BoylandEJ, HalfordJC (2013) Television advertising and branding. Effects on eating behaviour and food preferences in children. Appetite 62:236-241

110. Cairns G, Angus K, Hastings G, Caraher M (2013) Systematic reviews of the evidence on the nature, extent and effects of food marketing to children. A retrospective summary. Appetite 62:209-215

111. Ranciere F, Lyons JG, Loh VH, Botton J, Galloway T, Wang T et al (2015) Bisphenol A and the risk of cardiometabolic disorders: a systematic review with meta-analysis of the epidemiological evidence. Environ Health 14:46. doi:10.1186/ s12940-015-0036-5

112. Jordan S (2012) Monitoring für Prävention und Gesundheitsförderung. Präv Gesundheitsf 7(2):155-160

113. Geene R, Kliche T, Borkowski S (2015) Gesund aufwachsen: Lebenskompetenz, Bewegung, Ernährung im Setting Kita. Erfolgsabschätzung und Ableitung eines Evaluationskonzepts. Eine Expertise im Auftrag des Kooperationsverbundes gesundheitsziele.de. Gesellschaft für Versicherungswissenschaft und -gestaltung e.V. Geschäftsstelle gesundheitsziele.de, Köln, S29f 